Mitteilungen der Österreichischen Geographischen Gesellschaft, 159. Jg. (Jahresband), Wien 2017, S. 393-400

\title{
WEITERE INTERNATIONALE TOPONOMASTISCHE TAgUNGEN DES JAHRES 2017
}

\author{
Peter Jordan, Wien*
}

mit 4 Abb. im Text

Neben dem toponomastischen Hauptereignis des Jahres 2017, der 11. Konferenz der Vereinten Nationen zur Standardisierung geographischer Namen in New York, wartete das Jahr mit einer Fülle weiterer toponomastischer und onomastischer Tagungen auf.

Zwei von ihnen wurden von der Joint ICA/IGU Commission on Toponymy mitorganisiert. Dabei handelt es sich um ein Joint Venture der globalen Dachorganisationen von Kartographie und Geographie, der Internationalen Kartographischen Vereinigung (International Cartographic Association, ICA) und der Internationalen Geographischen Union (International Geographical Union, IGU). Es wurde im Jahr 2012 vom Verfasser mitbegründet - mit wesentlicher Unterstützung der damaligen Präsidenten der IGU, Ron ABLER, der ICA, Georg GarTnER, und der Expertengruppe der Vereinten Nationen für geographische Namen (United Nations Group of Experts on Geographical Names, UNGEGN), Helen Kerfoot. Die gemeinsame Kommission sollte in Ergänzung zur auf das Ziel der Standardisierung ausgerichteten UNGEGN und zum multidisziplinären International Council on Onomastic Sciences (ICOS) ein weltweites Forum für toponomastische Forschung speziell in den Wissenschaften Kartographie und Geographie bieten und damit solche Forschung fördern.

Die Leitung hatten von Anfang an als Chair von ICA-Seite Paulo DE Menezes (Brasilien) und als Chair von IGU-Seite Cosimo PaLAGiano (Italien) inne. Der Verfasser wurde bei der Internationalen Kartographischen Konferenz von Rio de Janeiro im Jahr 2015 offiziell zum gemeinsamen Vicechair bestellt und ist seit der Internationalen Kartographischen Konferenz von Washington D.C. im Juli 2017 Chair von ICA-Seite, während Paulo DE Menezes die Funktion des Common Vice-chair übernahm.

Die Kommission beteiligte sich seit ihrem Bestehen mit Sitzungen an zehn Kongressen und Konferenzen von ICA, IGU und der Vereinigung europäischer geographischer Gesellschaften (EuGeo) und richtete gemeinsam mit lokalen Veranstaltern und anderen Organisationen sieben spezielle Symposien aus. Von einigen erschienen auch Tagungsbände, die im Literaturverzeichnis genannt sind.

Im Folgenden soll nun kurz über fünf internationale zumindest teilweise toponomastische Ereignisse des Jahres 2017 berichtet werden. Diese Liste erhebt nicht den Anspruch, eine Aufzählung aller oder auch nur der wichtigsten Tagungen des Jahres auf diesem Gebiet zu sein, obwohl der ICOS-Kongress sicher zu letzteren zählt. Es handelt sich nur um jene Tagungen, an denen die Kommission (in zwei Fällen) oder der Verfasser (in allen Fällen) in irgendeiner Form (Mitorganisation, Sitzungsleitung, Vortrag) mitgewirkt haben.

\footnotetext{
* Hofrat Prof. h.c. Univ.-Doz. Dr. Peter JoRdan, Institut für Stadt- und Regionalforschung, Österreichische Akademie der Wissenschaften; Research Fellow, University of the Free State Bloemfontein, South Africa; Postgasse 7/4/2, A-1010 Wien; E-Mail: peter.jordan@oeaw.ac.at, http://www.oeaw.ac.at/isr
} 


\section{ICC Joint Pre-Conference Workshop: Charting the Cosmos of Cartography: History - Names - Atlases, Washington D.C. (USA), 28.-30. Juni 2017}

Drei Kommissionen der ICA hatten dieses zeitlich vor der Hauptkonferenz der ICA in Washington D.C. angesiedelte Symposion organisiert: die Commission on the History of Cartography (Chair: Imre Demhardt, USA), die ICA Commission on Atlases (vertreten durch Eric Losang, Deutschland) und die Joint ICA/IGU Commission on Toponymy (vertreten durch Peter JoRDAN). Es fand im Madison Building der Library of Congress (Abb. 1) nahe dem Kapitol statt. Die im gleichen Gebäude untergebrachte Kartensammlung der Library of Congress mit Prunkstücken der alten Kartographie wie der Waldseemüller-Karte aus 1507 bot reichlich Gelegenheit zu Besichtigungen, wurde von manchen Teilnehmern aber auch zu Forschungszwecken genützt.

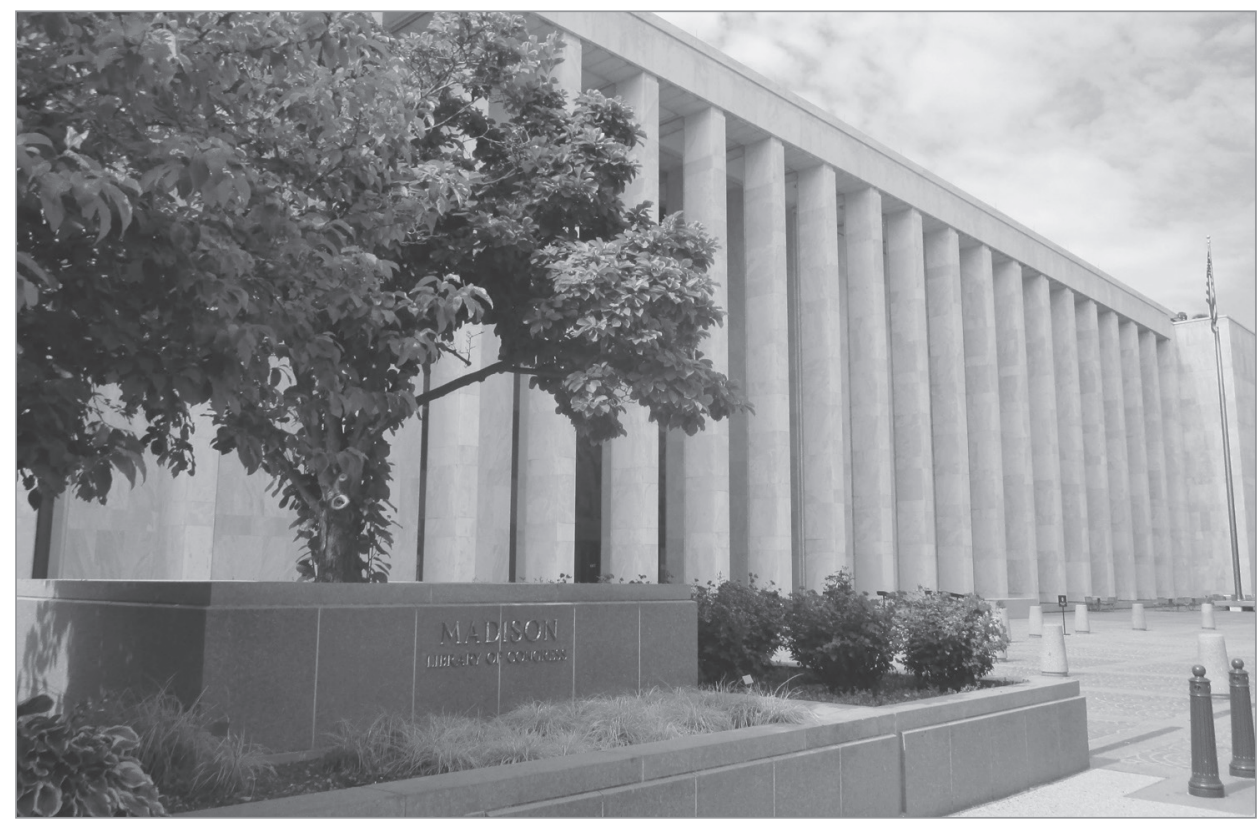

Foto: $\quad$ Peter JoRdAN 2017

Abb. 1: Madison Building der Library of Congress, Washington D.C.

Die drei Kommissionen hatten das Programm der Tagung paritätisch zusammengestellt, sodass die Geschichte der Kartographie, die Atlaskartographie und die Toponomastik in gleicher Weise mit Vorträgen und je einer Keynote zum Zug kamen. Der Programmablauf durchmischte die drei Bereiche aber bewusst, um einen Mehrwert durch inter(sub)disziplinären Austausch zu erzeugen. Tatsächlich ging dieser Plan auf, und es entwickelte sich eine rege Diskussion quer über die Teilgebiete hinweg. Auf Vorträge über die anderen beiden Teilgebiete von Kapazitäten wie Lindsay BraUN, Mark Monmonier, Imre Demhardt (alle USA), Lászlo Zentai (Ungarn), Jan Mokre (Österreich) zur Geschichte der Kartographie bzw. von Thomas Schulz (Schweiz), Eric Losang, Francis Harvey, Jana Moser (alle Deutschland) oder Igor DRECKI (Neuseeland) zur Atlaskartographie soll hier nur kurz hingewiesen werden. 
Für den toponymischen Teil sorgten Derek Alderman (USA), einer der Protagonisten der Kritischen Toponomastik, mit seiner Keynote über die Bedeutung der öffentlichen Repräsentanz ihrer Toponyme für das Selbstverständnis der Afro-Amerikaner; Peter JoRDAN mit einem Vortrag über die Wiedergabe von Minderheitennamen in den Blättern der Franzisco-Josephinischen Landesaufnahme unter besonderer Berücksichtigung der slowenischen und kroatischen Gebiete; Paulo de MeNEZES (Brasilien) mit einer vergleichenden Studie über die Namenschreibung in alten Karten der Provinz Rio de Janeiro; Elri LieBENBERg (Südafrika) mit einer Darstellung der Entwicklungsgeschichte einiger Toponyme in der südafrikanischen Kapprovinz auf der Grundlage alter Karten; Hyo Hyun Sung \& Sejin Ahn (Republik Korea) mit einer kritischen Analyse der Verwendung von generischen Bestandteilen in Namen von Meeren und Meeresteilen; Yaïves FerLand (Kanada) mit einem Vortrag über örtliche Systeme von Toponymen, also die gegenseitige Bezogenheit von geographischen Namen in einem bestimmten Gebiet.

Eine Auswahl der Vorträge wird im Cartographic Journal der British Cartographic Society veröffentlicht werden.

\section{Internationaler Kongress der onomastischen Wissenschaften, Debrecen (Ungarn), 27. August - 1. September 2017}

Der offiziell dreisprachige (Englisch, Französisch, Deutsch!) Internationale Rat der onomastischen Wissenschaften (International Council of Onomastic Sciences, ICOS) - in der Mehrheit doch linguistisch, aber die Multi- und Interdisziplinarität pflegend - veranstaltet alle drei Jahre seinen Weltkongress. Die Vorträge beziehen sich auf alle Arten von Eigennamen, also z.B. auch auf die Namen von Personen und Pflanzen. Geographische Namen spielten aber auf den letzten Kongressen eine zunehmende Rolle und waren auch diesmal ein wesentliches Thema. Allerdings war der Kongress mit 228 Teilnehmer weitaus kleiner als seine Vorläufer in Glasgow im Jahr 2014 und besonders in Barcelona im Jahr 2011. Das mag an der nicht so günstigen Erreichbarkeit des Tagungsortes Debrecen gelegen haben - besonders im Flugverkehr. Das Zentrum des ungarischen Calvinismus (Abb. 2) bot aber ein angenehmes Ambiente, besonders auch die ebenfalls calvinistische Universität der Stadt, deren Hauptgebäude der Tagungsort war.

Aus dem umfangreichen Tagungsprogramm können hier nur die Keynotes und die Österreich-Bezüge herausgehoben werden. In einer ersten Keynote ging Grant W. SмIтH (USA) auf die symbolische Kraft von Namen im Allgemeinen, aber besonders auch von geographischen Namen ein. Die zweite Keynote wurde vom schwedischen Linguisten und langjährigen Convenor der UNGEGN Working Group on Toponymic Terminology, Staffan Nyström, gehalten. Er sprach über die administrativen Zuständigkeiten für geographische Namen (Wer darf Ortsnamen vergeben?) und über damit verbundene Probleme und positive Beispiele. Als dritte Keynote schließlich boten István HoffMANN und Valéria TóTH von der örtlichen Universität, letztere auch die für die örtliche Organisation des Kongresses Hauptverantwortliche, einen Überblick über mögliche Typologien von Toponymen.

Die Teilnehmerzahl aus Österreich war trotz der relativen räumlichen Nähe Debrecens sehr überschaubar. Als Teil des regulären Programms hielten der Germanist Karl HoHEnsinNer (Adalbert-Stifter-Institut Linz) und der Slawist Hubert BERGMANN (Österreichische Akademie der Wissenschaften) einen Vortrag über die räumliche Verbreitung österreichischer Familiennamen und sprachen Florenza FisCHER und Holger Wochele (Wirtschaftsuniversität Wien) über „Deonymische Neubildungen als Globalismen in der Wirtschaftspresse: eine vergleichende Studie“.

Als Neueinführung im Rahmen der ICOS-Kongresse gab es diesmal innerhalb des Kongresses Symposien zu aktuellen Themen, zu denen jeweils vier Vortragende eingeladen worden wa- 


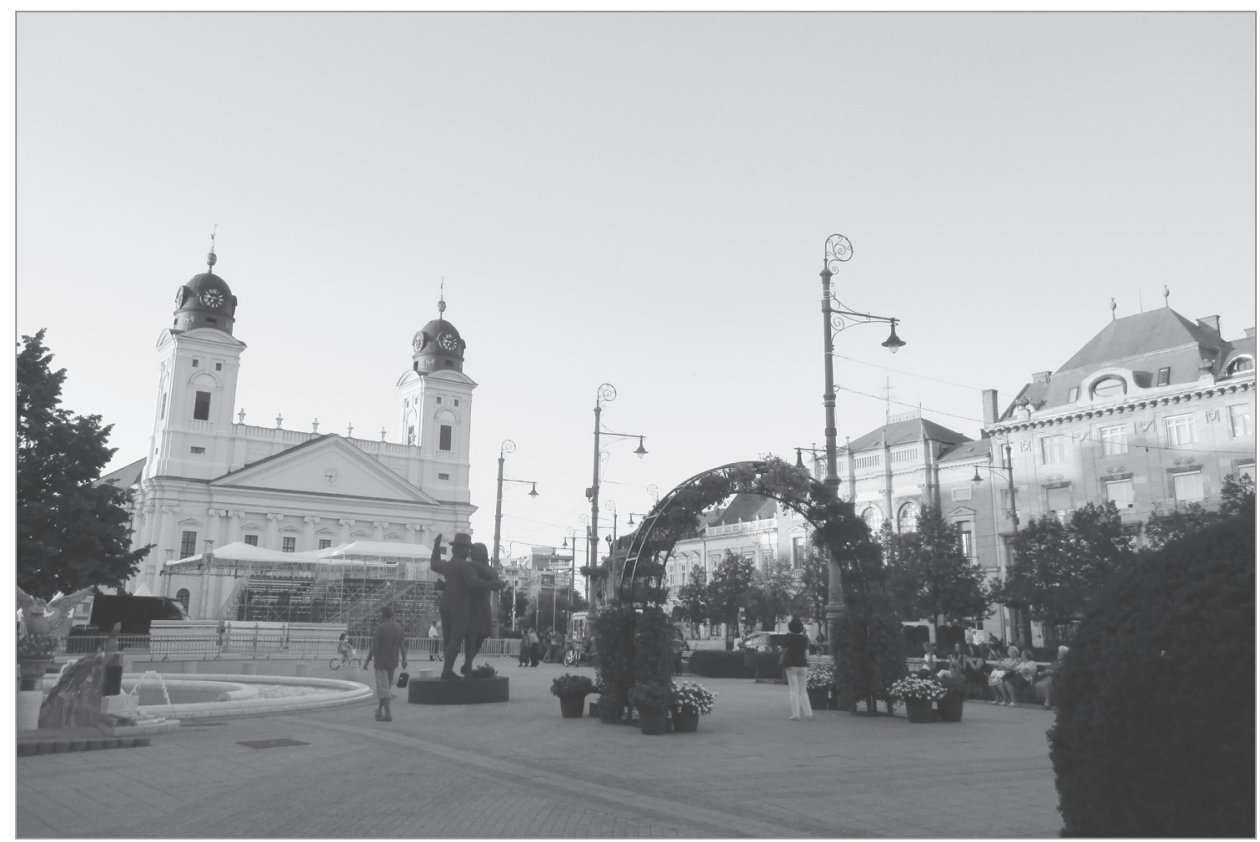

Foto: $\quad$ Peter JoRDAN 2017

Abb. 2: Das Zentrum von Debrecen mit der Reformierten Hauptkirche

ren. So sprach Peter JoRdAn im Rahmen des von Andrea BölCSKEI (Ungarn) und Oliviu FelecAN (Rumänien) organisierten und geleiteten Symposions zum Thema „Contacts of Name Systems in Central Europe“ zum Thema „The meaning of bi- or multilingual naming in public space for the cultural identity of linguistic minorities, demonstrated by examples from Central Europe”. Weitere Referenten dieses Symposions waren Paul Woodman (Vereinigtes Königreich), Andrea BölcSKeI und Christian Zschieschang (Deutschland).

Eine Auswahl der Vorträge wird in der Zeitschrift „Onomastica Uralica“ veröffentlicht werden.

\section{$4^{\text {th }}$ International Conference on Onomastics "Name and Naming" (ICONN 4), Baia Mare (Rumänien), 5.-7. September 2017}

Nur wenige Tage nach dem ICOS-Kongress in Debrecen konnte man an dieser kleinen onomastischen Tagung in der nahe gelegenen rumänischen Marmarosch [Maramureş] teilnehmen und wurde - wenn man wollte - sogar per Bus dorthin gebracht. Die durchaus große Stadt Baia Mare (124.000 Einwohner) ist bei uns wenig bekannt, aber bemerkenswert: Sie war eine historische Stadt, der man in der kommunistischen Periode eine nach sozialistischen Prinzipien gestaltete Neustadt gegenübergestellt hat. Baia Mare ist nach dem rumänischen Ortsnamengesetz von 2001 amtlich dreinamig und man kann auf den Ortstafeln neben dem rumänischen auch den ungarischen (Nagybánya) und den deutschen (Neustadt) Namen finden, wobei der deutsche Name bei uns kaum geläufig ist. 
In dieser Stadt fand nun im Abstand von jeweils zwei Jahren schon zum vierten Mal dieses immerhin von etwa 80 bis 100 Teilnehmern besuchte Symposion statt, das von Oliviu FeLECAN, Professor an der örtlichen Außenstelle der Technischen Universität Klausenburg [Cluj-Napoca] mit offensichtlich starker Unterstützung durch die örtlichen Behörden wieder vorzüglich organisiert war. Das Generalthema des Symposions „Das Sakrale und Profane in der Onomastik“ fand allerdings bei weitem nicht in allen Vorträgen seinen Niederschlag. Gheorghe CHIVU von der Rumänischen Akademie der Wissenschaften und Grant W. SмIтH (USA) hielten die Keynotes. Weitere prominente Vortragende waren Willi van LANGENDONCK (Belgien) und Luisa CAIAZzo (Italien). Aus Österreich gab es Vorträge von Holger Wochele, Peter Handler, Brigitte Seidler-Lunzer, Florenza Fischer (alle Wirtschaftsuniversität Wien). Der Verfasser hielt keinen Vortrag, war aber Mitglied des wissenschaftlichen Komitees und Leiter von zwei Sitzungen.

Für am östlichen Europa Interessierte war die Tagung wegen der vielen Teilnehmer aus diesen Ländern (neben Rumänien besonders Russland, Weißrussland und Polen) besonders ergiebig. Die Vorträge sollen in einem Tagungsband veröffentlicht werden.

\section{International Symposium on Place Names: Critical Toponymy - Place names in political, historical and commercial landscapes, Windhoek, Namibia, 18.-20. September 2017}

Das Symposion war von der Joint IGU/ICA Commission on Toponymy gemeinsam mit der University of the Free State (UFS) in Bloemfontein (Südafrika) und der University of Namibia (UNAM) organisiert worden und das zweite seiner Art nach jenem in Clarens (Südafrika) vor zwei Jahren. Die Veranstaltungsreihe soll zweijährlich fortgesetzt werden. Es handelt sich um eine Initiative des Department for Language Facilitation and Empowerment der University of the Free State mit den Promotoren Peter E. RAPER und Theodorus Du Plessis, und dem Ziel, der äußerst aktiven und forschungsintensiven onomastischen Szene des südlichen Afrikas eine Plattform zu bieten und länderübergreifenden Austausch zu ermöglichen.

Tatsächlich nahmen - wie auch schon am Symposion in Clarens - etliche Wissenschafter aus den Nachbarländern Südafrikas teil, obwohl das Gros wieder aus Südafrika kam. Das Einbeziehen der Joint ICA/IGU Commission soll den Einzugsbereich noch erweitern und auch außerafrikanische Aspekte ins Spiel bringen. Das ist bisher mit fünf bis acht Teilnehmern von außerhalb Afrikas erst teilweise gelungen. Vielleicht braucht es aber nur seine Zeit, bis die Attraktivität des für die Onomastik und besonders auch für die Toponomastik wegen seiner Sprachenvielfalt und seinen politischen Umbrüchen und der sich daraus ergebenden Fülle von Namen und Namensänderungen äußerst ergiebigen südlichen Afrikas zu wirken beginnt.

Gerade der Tagungsort Windhoek und Namibia sind dafür gute Beispiele. So schimmert die deutsche Kolonialzeit vor allem in den Städten noch stark durch: Obwohl das Englische die einzige offizielle Landessprache ist, finden sich in Windhoek und noch mehr in Swakopmund an der Atlantikküste oft vollständig (proprialer und generischer Bestandteil) deutsche Straßennamen (Abb. 3) und sind Geschäfte und auch alte Gebäude manchmal ausschließlich auf Deutsch beschriftet (Abb. 4).

Die Tagung selbst gewährte einen guten Einblick in die reiche onomastische Forschung des südlichen Afrikas. So gingen Matthias BrEnZINGER und Peter E. RAPER in ihren Keynotes auf Namen in den Khoisansprachen mit ihren Klicklauten ein und stellten Lucie A. MöLler und Berthie Netheling ihre neuen Bücher „The Dictionary of Southern African Place Names“ bzw. „What's in a name?“ vor. Aus Europa trugen Drago KLADNIK und Matjaž GERŠIČ (Slowenien) mit einem Überblick über die Verwendung slowenischer geographischer Namen in der Werbung, Cosimo Palagiano (Itali- 


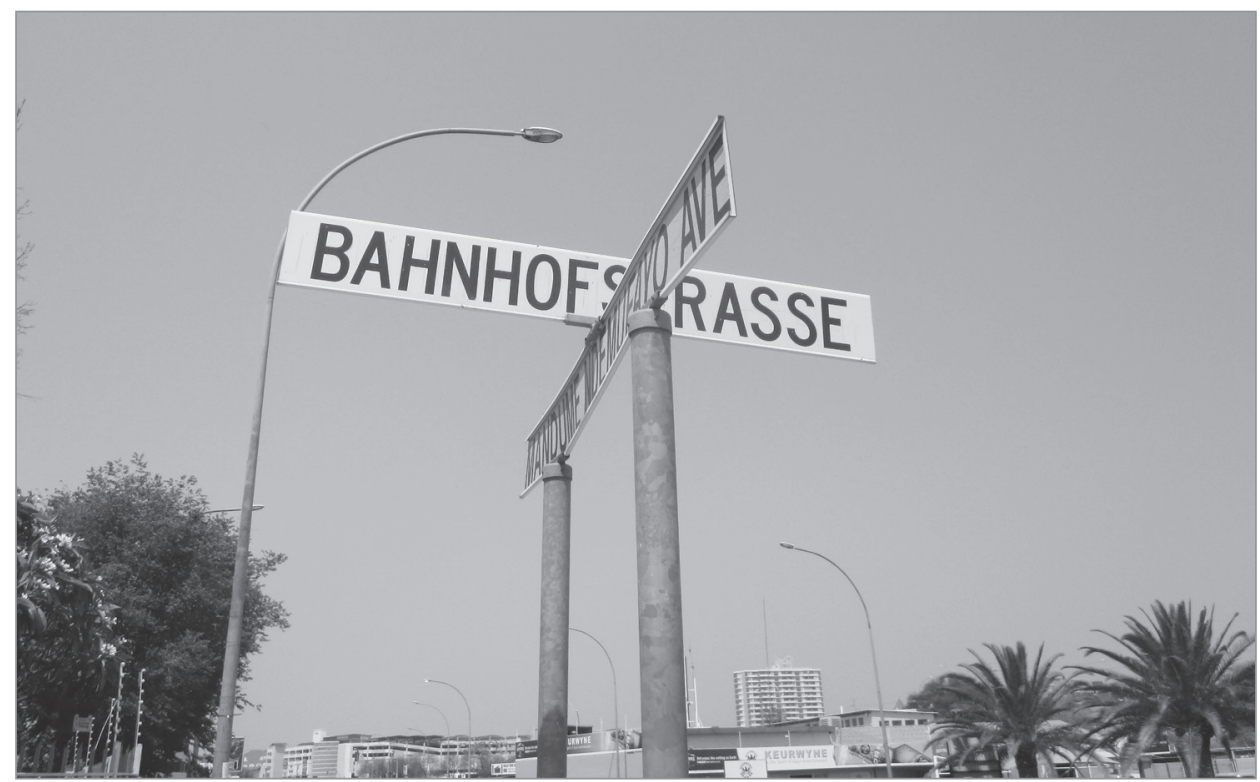

Foto: $\quad$ Peter Jordan 2017

Abb. 3: Deutsches Straßenschild in Windhoek, Namibia

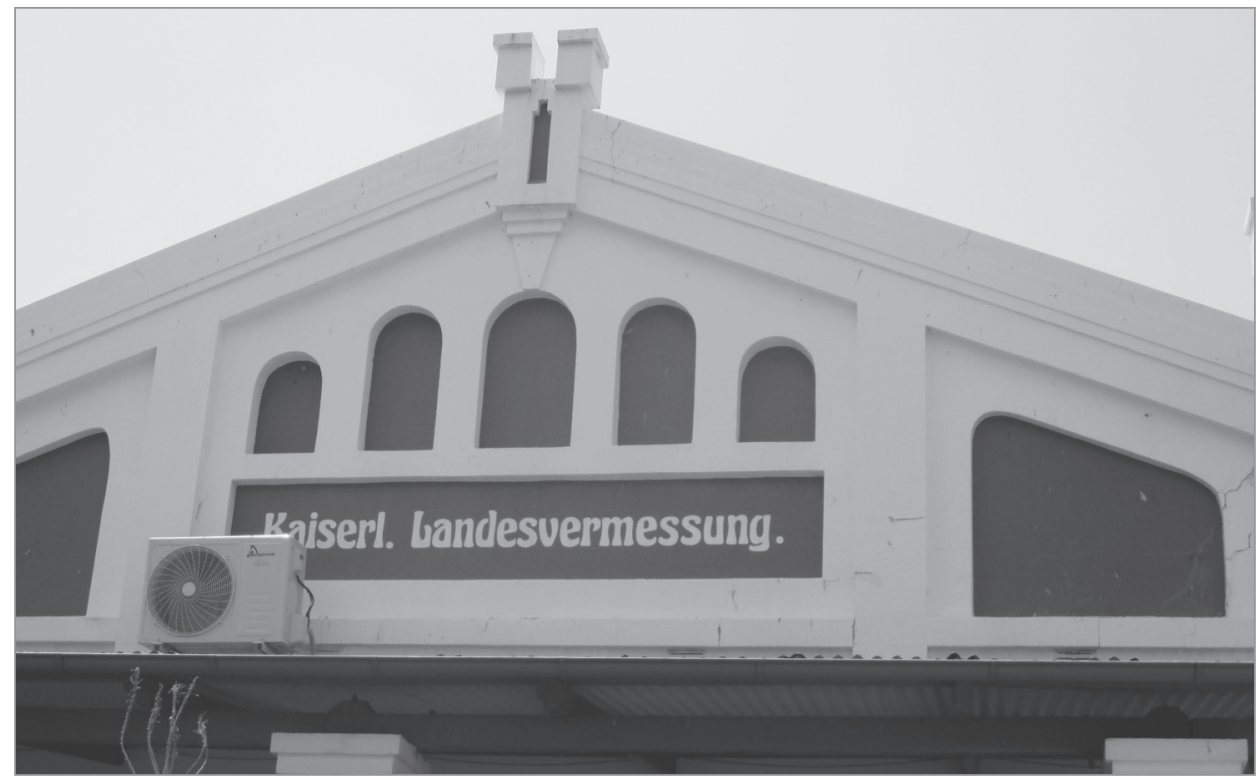

Foto: $\quad$ Peter JORDAN 2017

Abb. 4: Gut erhaltenes Gebäude der Kaiserlichen Landesvermessung mit deutscher Aufschrift in Windhoek, Namibia 
en) über die kommerzielle Verwendung geographischer Namen, Günther SchUPPENER (Deutschland, derzeit Universität Tyrnau [Trnava], Slowakei) über Mehrnamigkeit als Problem historischer Forschung und der Verfasser mit dem Versuch einer Typisierung der kommerziellen Verwendung geographischer Namen, untermalt von Beispielen aus Österreich, zum Tagungsprogamm bei.

Eine Auswahl der Vorträge soll in der südafrikanischen onomastischen Zeitschrift „Nomina Africana" veröffentlicht werden.

\section{$2^{\text {rd }}$ International Seminar on Sea Names, Berlin, 22.-25. Oktober 2017}

Die Republik Korea unternimmt außerordentliche Anstrengungen, die Länder und Sprachgemeinschaften der Welt davon zu überzeugen, dass zur Bezeichnung des Meeres zwischen der koreanischen Halbinsel und dem japanischen Archipel neben dem jeweiligen Äquivalent für Japanisches Meer auch noch Ostmeer verwendet werden soll. Es kann dies als das Empanzipationsbedürfnis eines jungen Staates gegenüber dem früheren regionalen Hegemon Japan gedeutet werden. Sie unterhält dazu einen eigenen Botschafter für geographische Namen, ist auf internationalen Tagungen der Toponomastik (besonders jenen der Vereinten Nationen und anderer internationaler Organisationen) stets zahlreich und überaus aktiv vertreten, betreibt Gesellschaften wie die Society for East Sea und die Northeast Asian History Foundation, welche die Verbreitung des Namens Ostmeer auf ihre Fahnen geheftet haben, und veranstaltet jährlich und nun schon zum 23. Mal internationale Seminare in verschiedenen Teilen der Welt, um dieses Anliegen zu unterstützen.

$\mathrm{Zu}$ einer großen Teilnehmerschaft aus der Republik Korea und einer größeren Zahl von Mitwirkenden aus dem jeweiligen Gastgeberland werden dazu immer auch Wissenschafter aus Japan und namhafte Toponomasten aus anderen Teilen der Welt eingeladen. Natürlich ist der Name dieses Meeres das Kernthema, doch werden durchaus auch andere toponomastische Fragen diskutiert und verhalten sich die Teilnehmer keineswegs einhellig dem koreanischen Anliegen gewogen. Gerade die internationalen Experten äußern oft kontroversielle Ansichten und fordern die Proponenten des Ostmeer-Gedankens mit ihren Argumenten heraus. Es handelt sich also immer auch um wissenschaftlich wertvolle Veranstaltungen.

So war auch das diesjährige Seminar wieder von der Society for East Sea und der Northeast Asian History Foundation, in Person maßgeblich von Sungjae Сноо, der auch UNGEGN-Experte und UNGEGN Convenor ist, organisiert. Als örtlichen Mitveranstalter hatte man das Geographische Institut der Humboldt-Universität zu Berlin, vertreten durch Péter BAGolY-Simó, gewonnen. Unter den namhaften internationalen Experten, die Vorträge hielten, wären besonders Helen KERFоOт (Kanada), die frühere Vorsitzende der UNGEGN, Paul Woodman (Vereinigtes Königreich), früherer Vertreter seines Landes in der UNGEGN und besonders auf dem Gebiet der Exonymie ausgewiesen, Joseph SтоLTMAN (USA), einer der führenden geographischen Fachdidaktiker seines Landes, Brahim Atoui (Algerien), Leiter des UNGEGN Task Team for Africa, Peder Gammeltoft (Dänemark), neuer Convenor der UNGEGN Working Group on Toponymic Training, und Kohei Watanabe (Japan), neuer Convenor der UNGEGN Working Group on Exonyms, zu nennen. Aus Österreich hielten der Koreanist an der Universität Wien Rainer DoRmels, die frühere Vorsitzende des österreichischen Expertengremiums für geographische Namen (AKO) Isolde HAUSNER sowie der Verfasser Vorträge. DoRmels und der Verfasser fungierten auch als Leiter von Sitzungen.

Als Fazit kann festgehalten werden, dass sich der Name Ostmeer tatsächlich - allein schon als Folge der Aktivitäten der Republik Korea - verbreitet, in erster Linie in Kombination mit Japanisches Meer etwa in der Form Japanisches Meer/Ostmeer, dass aber kontextabhängig auch die Verwendung nur eines Namens, dann wohl in erster Linie von Japanisches Meer, möglich sein müsse und dass es prinzipiell im Ermessen der Empfängersprachen und Empfängerländer liege, ein 
bestimmtes Exonym zu verwenden. Wie auch in anderen Fällen (z.B. Côte d'Ivoire) sei es nicht das Geberland, das den Exonymengebrauch bestimmen könne.

Als Resümee dieser toponomastischen Tagungen des Jahres 2017 kann aus der Sicht eines kulturgeographischen Toponomasten festgehalten werden:

- Geographische Namen sind zu einem wichtigen Thema der Kulturgeographie geworden und Geographen schalten sich zunehmend in die toponomastische Diskussion ein.

- Die Kritische Toponomastik im Sinne eines Ausleuchtens des politischen und gesellschaftlichen Hintergrundes von geographischen Namen und des Umgangs mit ihnen ist heute der vielleicht stärkste Strang der kulturgeographischen Sichtweise auf geographische Namen.

- Wichtige und häufig behandelte Themen sind in diesem Bereich geographische Namen als symbolische Ebene tieferliegender gesellschaftlicher Konflikte oder der Umgang mit Namen sprachlicher Minderheiten als Ausdruck interkultureller Beziehungen.

- Viel Beachtung wird auch der Kommerzialisierung der Namenlandschaft und der kommerziellen Verwendung geographischer Namen geschenkt. Dabei stellt sich die Frage, inwieweit bodenständige geographische Namen nicht ein Teil des kulturellen Erbes sind und deshalb des Schutzes bedürfen.

\section{Literaturverzeichnis}

Da Rocha Bérenger Resende A.C., Jeney J. (Hrsg.), Symposion on Atlases, Toponymy and the History of Cartography. Proceedings of the ICC 2015 Pre-Conference Rio de Janeiro, Brazil, 20-21 August 2015. Sine loco [Rio de Janeiro], ICA International Cartographic Association.

Jordan P., Woodman P. (Hrsg.) (2016), Place-Name Changes. Proceedings of the Symposion in Rome, 17-18 November 2014 (= Name \& Place, 5). Hamburg, Verlag Dr. Kovač.

Moser J. (Hrsg.) (2016), Joint Commission Seminar on Historical Maps, Atlases and Toponymy (= forum IfL, 30). Leipzig, Leibniz-Institut für Länderkunde.

Nomina Africana, Journal of the Names Society of Southern Africa, 30, 1, 2016: special issue containing 10 papers presented at the Symposion "Place names, diversity and heritage", Clarens, Free State, South Africa, 17-18 September 2015.

Semestrale di Studi e Ricerche di Geografia, XXV, 2, Roma 2013: Special issue including toponymy papers presented at the IGU Regional Conference Kyoto, Japan, 4-9 August 2013. - http:// www.semestrale-geografia.org/index.php/sdg/issue/view/3 